\title{
Multi-functional Resin Coated Sand Proppants: Examination using Microscopy and Energy Dispersive X-ray Spectroscopy
}

\author{
David Reuschle $^{1 *}$, Arjun Raghuraman ${ }^{2}$ and Ann Johnson ${ }^{1}$ \\ 1. The Dow Chemical Company, Core R\&D Analytical Science, Lake Jackson, TX USA. \\ 2. The Dow Chemical Company, Polyurethanes Process R\&D, Lake Jackson, TX USA. \\ * Corresponding author: dreuschle@dow.com
}

The process of hydraulic fracturing is used in enhanced recovery of oil and gas wells. In the case of shale gas wells, hydraulic fracturing is conducted by pumping bore fluid into a shale formation. The elevated pressure causes the shale to develop cracks that allow trapped hydrocarbons to move into the well. Solid particulates are often added to the bore fluid in order to "prop" the newly formed cracks open. These solid particulates are called proppants. The composition of the bore fluid is generally water $(90-95 \%)$, proppant $(5-10 \%)$ and other chemicals $(<1 \%)$. Some of the proppants used in these applications include ceramics, bare silica sand, and resin coated sand (RCS) [1]. This paper is concerned with RCS proppants only.

Primary performance characteristics of the RCS proppants include increased crush strength (relative to bare sand), minimization of fines formation due to the resin coating, and blocking of neighboring RCS particles thereby preventing back-flow of the proppant into the production fluid. Secondary performance characteristics can be imparted to the RCS by altering the formulation of the coating [2]. An example of a secondary performance characteristic would be the incorporation of an inorganic salt to the resin coating, allowing for selective capture of radium isotopes [3]. The use of capture agents allows for better wastewater management and protection of surface and ground water, enabling more successful and sustainable operation of wells [4]. Radium is particularly problematic because it is soluble in water and can travel to the surface resulting in equipment contamination. Radium contamination can introduce additional challenges in the disposal and re-use of wastewater.

This study utilizes a combination of electron microscopy and X-ray spectroscopic techniques to understand the thickness of the resin coating, the consistency of the resin coating, and the location of the inorganic salt capture agent within the resin coating. Because the precipitation of radium from brine occurs either by lattice replacement or adsorption, it is important that the capture agent be exposed at the surface of the resin coating [5]. Formulation of the RCS containing an inorganic salt was optimized to produce coated sand with good primary characteristics and useful secondary characteristics. The formulation development work is out of scope for this paper. The primary performance characteristics will be a function of the coating material, coating thickness and distribution. The secondary performance characteristics will be a function of the coating material, the location of the capture agent within the resin coating, and the composition of the produced water and the downhole conditions.

Four low magnification backscatter electron images are shown in Figure 1. The location of the inorganic salt is indicated by the bright patches on the sand. The dark patches on the sand indicate areas where only resin is present. Overall, the coverage of resin is good - some areas of the sand are covered with inorganic salt particles. The sand grain with the least coverage appears to be approximately half covered with the capture agent particles. Higher magnification images of the RCS surface show the particles embedded in the resin coating. Some of the capture agent particles show evidence of a thin 
resin coating obscuring the lower portion of the particle, and some of the particles appear to be buried well beneath the surface of the resin. The EDS spectra in Figure 1 show an overlay of the capture agent in the resin coating and pure inorganic salt as a powder. The slightly lower X-ray signal observed for both $\mathrm{S}$ and $\mathrm{O}$ in the resin coated sample suggests that a thin coating of resin is covering the capture agent particles in the RCS. The slightly greater X-ray signal for C suggests the same. These results indicate that the inorganic salt particles were coated with resin during the production of the RCS, and that capture agent particles are not exposed directly at the surface.

\section{References:}

[1] Montgomery, C.T. and Smith, M.B. J. Pet. Technol. 62 (2010), p. 26-28, 30-32.

[2] Duenckel, R. J., Leasure, J. G. and Palisch, T. SPE Hydraulic Fracturing Technology Conference (2014) doi:10.2118/168605-MS.

[3] Goyal, S., et al. SPE Hydraulic Fracturing Technology Conference and Exhibition (2017) doi:10.2118/184853-MS.

[4] Lutz, B.D., Lewis, A.N., and Doyle, M.W. Water Resour. Res. 49 (2013), p. 647-656.

[5] Zhang, T. et al. Environ. Sci. Technol. 48 (2014), 4596-4603.

[6] The authors acknowledge Sachit Goyal for providing the materials and expert discussion in these analyses.
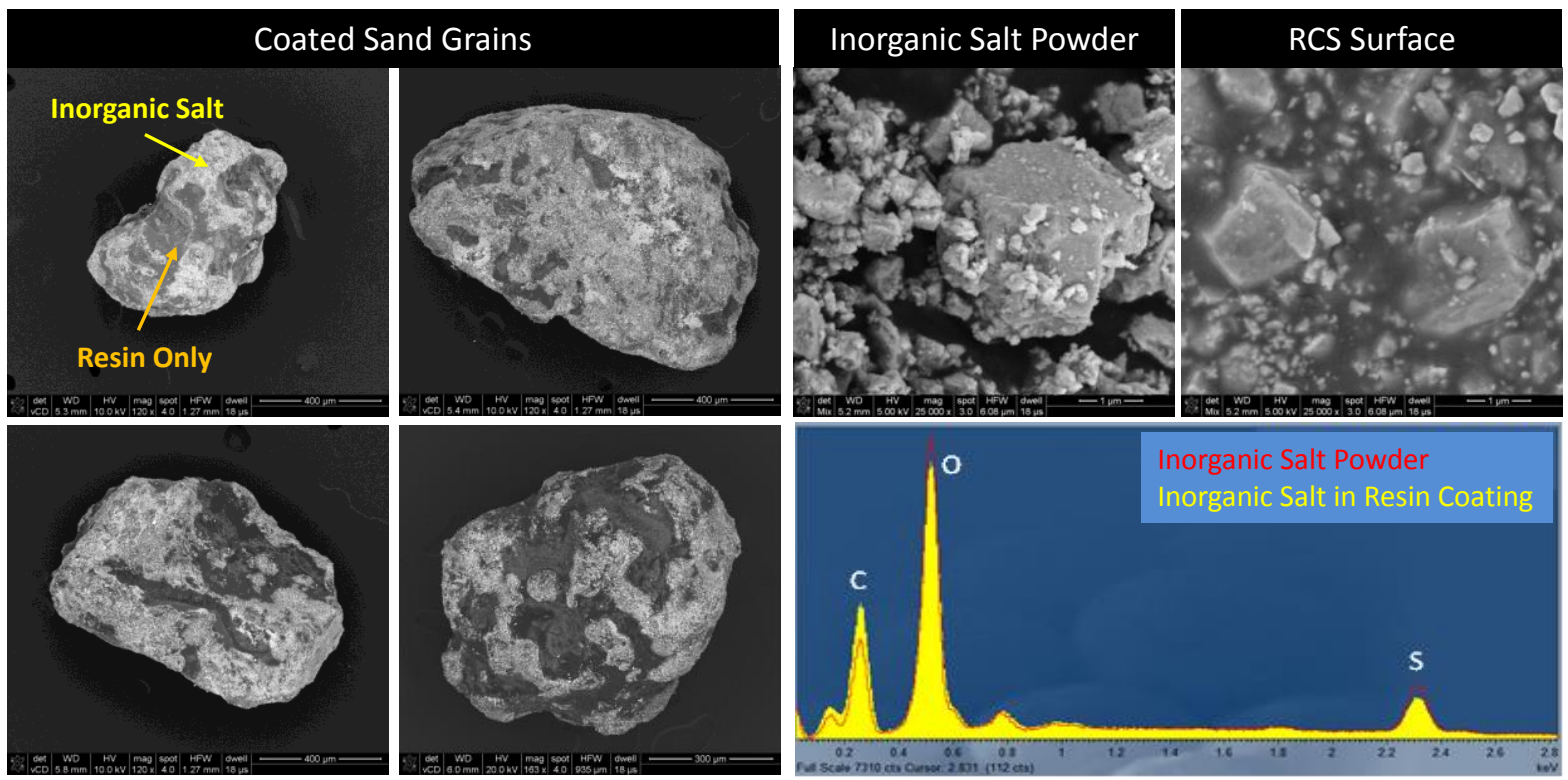

Figure 1. Top down backscatter electron images of resin costed sand containing inorganic particles. The intensity of oxygen and sulfur are slightly attenuated in the spectrum of the resin coating. 